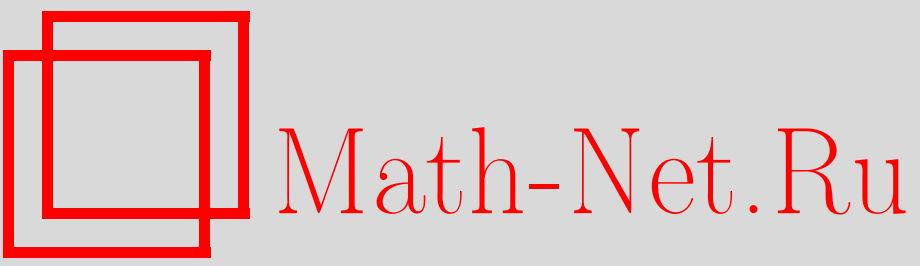

А. М. Вершик, Н. А. Сидоров, Биективные кодирования автоморфизмов тора и бинарные квадратичные формы, УМН, 1998, том 53, выпуск 5, 231-232

DOI: https://doi.org/10.4213/rm86

Использование Общероссийского математического портала Math-Net.Ru подразумевает, что вы прочитали и согласны с пользовательским соглашением

http://www.mathnet.ru/rus/agreement

Параметры загрузки:

IP: 54.237 .206 .68

26 апреля 2023 г., 13:45:15 


\title{
БИЕКТИВНЫЕ КОДИРОВАНИЯ АВТОМОРФИЗМОВ ТОРА И БИНАРНЫЕ КВАДРАТИЧНЫЕ ФОРМЫ
}

\author{
А. М. ВеРшик, Н. А. Сидоров
}

Рассматриваются специальные отображения некоторого символического компакта на двумерньй тор, сопрягающие двусторонний сдвиг с заданным гиперболическим автоморфизмом двумерного тора и сохраняющие меру максимальной энтропии. В отличие от известного геометрического метода кодирования при помощи марковских разбиений (см. ссылки в [2], [9]) рассматриваемое кодирование исползует арифметическую и групповую структуру. Это позволяет связать ариффметику соответствуюшего алгебраического расширения $\mathbb{Q}$ и свойства некоторой бинарной квадратичной формы с динамикой автоморфизма. Указанные отображения параметризуются единицами квадратичного поля или же гомоклиническими точками, лежащими на некоторой выделенной орбите.

Пусть $T$ - гиперболический автоморфизм $\mathbb{T}^{2}$, задаваемый матрицей $M=\left(\begin{array}{ll}a & b \\ c & d\end{array}\right) \in G L(2, \mathbb{Z})$. Положим $r=\operatorname{Tr} M, \sigma=\operatorname{det} M, D=r^{2}-4 \sigma$. Из гиперболичности следует, что $r \neq 0$ для $\sigma=-1$, и $|r| \geqslant 3$ для $\sigma=+1$. Для простоты будем считать, что $r>0$. Пусть $\lambda=(r+\sqrt{D}) / 2-$ болшшее собственное значение $M$. В качестве символического компакта $\mathfrak{X}$ выберем либо стационарный марковский компакт $\mathfrak{X}_{r}$ с пространством состояний $0,1, \ldots, r$ и попарными запретами $\left\{\varepsilon_{n}=r \Rightarrow \varepsilon_{n+1}=0, n \in \mathbb{Z}\right\}$, если $\sigma=-1$, либо софический компакт $\mathfrak{Y}_{r}=\left\{\left\{\varepsilon_{n}\right\}_{-\infty}^{\infty}\right.$ : $0 \leqslant \varepsilon_{n} \leqslant r-1,\left(\varepsilon_{n} \ldots \varepsilon_{n+s}\right) \neq(r-1)(r-2)^{s-2}(r-1)$ для любого $n \in \mathbb{Z}$ и любого $\left.s \geqslant 2\right\}$ для $\sigma=+1$. Каждый из двух компактов, таким образом, полностью определяется следом и является $\beta$-жомпактом для $\beta=\lambda$ (см. [7]). Пусть $\tau$ обозначает двусторонний сдвиг на $\mathfrak{X}$, т.е. $(\tau \varepsilon)_{n}=\varepsilon_{n+1}$, и $\mu$-мера максимальной энтропии. Определим операцию нормализации как отображение $\mathfrak{n}: \prod_{-\infty}^{\infty}\{0,1, \ldots, 2 r\} \rightarrow \mathfrak{X}$ следующим образом: если последовательность $\left\{x_{k}\right\}$ финитна, то $\mathfrak{n}\left(\left\{x_{k}\right\}\right)=\left\{\varepsilon_{k}\right\}_{l}^{l^{\prime}}-$ последователшность коэффициентов марковского (софического) $\beta$-разложсния с $\beta=\lambda$ числа $x=\sum_{k} x_{k} \lambda^{-k}$; точнее, $l=\left[-\log _{\lambda} x\right]+1, \varepsilon_{l}=\left[\lambda^{l} x\right]$, $\varepsilon_{k}=\left[\lambda S^{k-l}\left(\lambda^{l} x\right)\right], k>l$, где $S x=\{\lambda x\}$. Для произвольной последовательности нормализация определяется как слабый предел нормализаций финитных аппроксимаций. По поводу корректности определения см. [4], [9]. Сумма двух последовательностей из $\mathfrak{X}$ определяется как нормализация их покоординатной суммы, аналогично определяется обратньй элемент по сложению. Можно показать, что что сумма и разность определены для п.в. пар последователшностей по мере $\mu \times \mu$. После естественной арифметической факторизации mod 0 по мере максимальной энтропии $p: \mathfrak{X} \rightarrow \mathfrak{X}^{\prime}$ на фактор-множестве $\mathfrak{X}^{\prime}$ определяется групповая структура, относительно которой сдвиг $\tau$ есть групповой автоморфизм.

Напомним, что точка $\mathbf{s} \in \mathbb{T}^{2}$ назывется гомоклиничной нулю (кратко: гомоклинической), если она лежит в пересечении слоев расширяющегося и сжимающегося слоений, проходящих че-

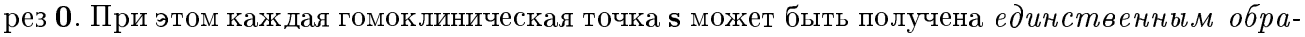
зом как проекция на собственную прямую $L u$, отвечающую собственному значению $\lambda$, вдоль второй собственной прямой $L_{s}$, некоторой точки решетки $\mathbf{z}(\mathbf{s}) \in \mathbb{Z}^{2}$ и последующем проектировании полученной точки $\mathbf{t}=\mathbf{t}(\mathbf{s})$ на тор (см. [1]). Точку $\mathbf{t}=\mathbf{t}(\mathbf{s})$ будем назьвать плоской координатой гомоклинической точки $\mathbf{s}$.

Лемма 1. Плоская координата любой гомоклинической точки $T$ имеет следующ,ий вид: $\mathbf{t}(\mathbf{s})=\mathbf{t}(u, v)=((n+u \lambda) / \sqrt{D},(k+v \lambda) / \sqrt{D})$, əде $(u, v)=\mathbf{z}(\mathbf{s}), u\left(\begin{array}{c}n \\ k\end{array}\right)=-\sigma M^{-1}\left(\begin{array}{l}u \\ v\end{array}\right)$.

ОПредЕЛЕНИЕ. Арифметическим кодированием автоморфизма $T$ называется сквозное отображение $\mathfrak{X} \rightarrow \mathfrak{X}^{\prime} \rightarrow \mathbb{T}^{2}$, где первое отображение есть факторизация $p$ (см. выше), а второе - групповой гомоморфиим $\mathfrak{X}^{\prime} \rightarrow \mathbb{T}^{2}$, переводящий сдвиг $\tau$ в $T$.

Теорема 2. Любое арифметическое кодирование гиперболического автоморфизма Т имеет вид

(1)

$$
\varphi_{\mathbf{s}}(\varepsilon)=\lim _{N \rightarrow+\infty}\left(\left(\sum_{-N}^{N} \varepsilon_{n} T^{-n} \mathbf{t}(\mathbf{s})\right) \bmod \mathbb{Z}^{2}\right)=\lim _{N \rightarrow+\infty}\left(\left(\sum_{-N}^{N} \varepsilon_{n} \lambda^{-n}\right) \mathbf{t}(\mathbf{s}) \bmod \mathbb{Z}^{2}\right),
$$


где $\mathbf{s}$ - ненулевая гомоклиническая точка $T, \mathbf{t}(\mathbf{s})$ - ее плоская координата, а умножение под знаком суммы понимается как умножсние числа на вектор в $\mathbb{R}^{2}$.

ОПРЕДЕлЕниЕ. Бинарная квадратичная форма $f_{T}(x, y)=c x^{2}+(a-d) x y-b y^{2}$ называется формой, ассоцированной с автоморфизмом $T$.

Теорема 3. Отображение $\varphi_{\mathbf{S}}(\cdot)$ переводит меру $\mu$ в меру Хаара на торе. При этом число прообразов почти всякой точки равно $K$, где $K=\left|f_{T}(u, v)\right|, a(u, v)=\mathbf{z}(\mathbf{s})$.

Если $\varphi_{\mathbf{s}}$ - биекция п.в., мы будем называть такое кодирование биективным арифметическим кодированием (БАК). Оказывается, БАК существуют далеко не для всех автоморфизмов $T$.

Теорема 4. 1. Следующие предложения әквивалентны:

(а) Гиперболический автоморфизм $T$, задаваемый матрицей $M$, допускает биективное арифметическое кодирование.

(б) $M$ алгебраически сопряжена в $G L(2, \mathbb{Z})$ союзной матрище $C_{r, \sigma}=\left(\begin{array}{cc}r & 1 \\ -\sigma & 0\end{array}\right)$, где $r=\operatorname{Tr} M u \sigma=\operatorname{det} M$.

(в) Разрешимо диофантово уравнение

$$
f_{T}(x, y)= \pm 1 \text {. }
$$

(г) Существует точка $(x, y) \in \mathbb{Z}^{2}$, линейная оболочка орбить которой под действием степеней $M$ есть $\mathbb{Z}^{2}$. При этом любая такая точка удовлетворяет уравнению (2).

2. Пусть Т допускает БАК, и $\lambda \neq \frac{3+\sqrt{5}}{2}$. Существует естественное взаимно-однозначное соответствие между множеством биективных арифметических кодирований $T$, группой Дирихле $\mathscr{U}(T)=\{A: A T=T A\}=\left\{ \pm T^{k} \mid k \in \mathbb{Z}\right\}$ и әруппой единии, $\mathscr{U}(D)=\left\{ \pm \lambda^{k} \mid k \in \mathbb{Z}\right\}$ квадратичного поля $\mathbb{Q}(\sqrt{D})$. А именно: если $\varphi_{\mathbf{s}_{1}}, \varphi_{\mathbf{s}_{2}}-$ два БАK, то $\varphi_{\mathbf{s}_{2}}=A \varphi_{\mathbf{s}_{1}}$ для некоторого $A \in \mathscr{U}(T), u \mathbf{t}\left(\mathbf{s}_{2}\right)=\delta \mathbf{t}\left(\mathbf{s}_{1}\right)$, где $\delta \in \mathscr{U}(D)$.

В случае, когда $M$ не сопряжена союзной матрице, аналогом БАК является минимальное арифметическое кодирование, определяемое как кодирование, имеющее наименьшее возможное число прообразов $m$. Из теоремы 3 вытекает, что $m=\min \left\{\left|f_{T}(u, v)\right|:(u, v) \in \mathbb{Z}^{2} \backslash(0,0)\right\}$, т.е. $m$ есть арифметический минимум квадратичной формы $f_{T}$.

Идеи символического кодирования автоморфизмов тора при помощи арифметических разложений возникли в работе [1], по другому поводу рассматривались в [3], обсуждались и развивались в [5], [6], [8]-[10].

Часть резултатов может быть обобщена на гиперболические автоморфизмы многомерных торов. В последующих работах этот случай будет рассмотрен подробно.

Доказательства утверждений настоящей статьи можно найти в полной версии [9].

\section{СПИСОК ЛИТЕРАТУРЫ}

[1] Вершик А. М. // Функц. анализ и его прил. 1992. T. 26. С. 22-24. [2] Adler R. L. // Bull. Amer. Math. Soc. 1998. V. 35. P. 1-56. [3] Bertrand-Mathis A. // Bull. Math. Soc. Fr. 1986. V. 114. P. 271-323. [4] Frougny Ch., Sakarovitch J. (to appearInternat. J. Algebra Comput). [5] Kenyon R., Vershik A. // Ergodic Theory Dynam. Systems. 1998. V. 18. P. 357-372. [6] Le Borgne S. Dynamique symbolique et propriétés stochastiques des automorphisms du tore: cas hyperbolique et quasi-hyperbolique // Thèse doctorale, 1997. [7] Parry W. // Acta Math. Hungar. 1960. V. 11. P. 401-416. [8] Sidorov N. A., Vershik A. M. Ergodic properties of Erdös measure, the entropy of the goldenshift, and related problems // Preprint IMS 14/96; to appear in Monatsh. Math. [9] Sidorov N. A., Vershik A. M. Bijective arithmetic codings of hyperbolic automorphisms of the 2-torus, and binary quadratic forms // Preprint IML 98/21; to appear in J. Dynam. Control Sys. [10] Einsiedler M., Schmidt K. // Труды МИРАН. 1997. V. 216. Р. 259-279.

Санкт-Петербургское отделение

математического института им. В. А. Стеклова РАН

Принято редколлегией

E-mail: vershik@pdmi.ras.ru sidorov@pdmi.ras.ru

19.08.1998 